\title{
Epidemiological Differences of COVID-19 Over the World
}

\author{
Abida Sayed ${ }^{1}$, Krishna Teja Challa ${ }^{1}$, Sateesh Arja ${ }^{2}$ \\ 1. Department of Medicine and Research, Avalon University School of Medicine, Willemstad, CUW 2. Medicine/Clinical \\ Skills, Avalon University School of Medicine, Willemstad, CUW
}

Corresponding author: Sateesh Arja, sarja@avalonu.org

\section{Abstract \\ Background}

Coronavirus disease 2019 (COVID-19), originally, from Wuhan, China, has now spread to most countries across the globe and devastated global healthcare systems. The impact of this disease has, however, shown baffling variations in prevalence in different regions of the world. The aim of this short review is to identify differential national COVID-19 prevalence of COVID-19, as well as to suggest these epidemiological differences.

\section{Methods}

A review of studies was conducted using PubMed and Google Scholar search engines. Search tactics were centered on COVID-19 (“COVID-19" AND “coronavirus”) and BCG vaccination ("BCG vaccination” OR “Bacillus Calmette-Guérin” OR “vaccine”)

\section{Results}

It is found that national prevalence differences may be linked with BCG childhood immunization history. A statistically insignificant difference was observed in COVID-19 prevalence when comparing countries with a BGC policy and countries without it $(\mathrm{P}>0.05)$. This inconclusivity suggests the influence of confounders in this study.

\section{Conclusions}

National differences in COVID-19 cases can be attributable to immunologic regulations, such as BCG vaccination protocols. Caution should be taken in establishing a correlation between COVID-19 prevalence and BCG vaccination, partly due to the weak quality of statistical data on COVID-19 related to poor testing rates in countries with BCG vaccination policy. Nonetheless, the analysis of the epidemiological aspects of COVID-19 will shed light on future efforts towards effective control and prevention.

Received 07/08/2020

Review began 07/09/2020 Review ended 09/06/2020 Published 09/08/2020

\section{๑) Copyright 2020}

Sayed et al. This is an open access article distributed under the terms of the Creative Commons Attribution License CC-BY 4.0., which permits unrestricted use, distribution, and reproduction in any medium, provided the original author and source are credited.
Categories: Internal Medicine, Infectious Disease, Epidemiology/Public Health Keywords: coronavirus, covid-19, bcg vaccine, bacillus calmette guerin vaccine

\section{Introduction}

A novel coronavirus referred to as coronavirus disease 2019 (COVID-19) or severe acute respiratory syndrome 2 (COVID-19 or SARS-COV2) continues to devastate the world. Currently, there are a total number of 26,795,708 confirmed COVID-19 cases recorded with 878,963 deaths worldwide as of 09-05-2020 [1]. The exponential growth in case numbers has shaken the healthcare and economic systems worldwide with alarming regional discrepancies being observed [2]. Eastern regions with relatively fragile healthcare systems show lower than expected incidence and mortality rates [3]. Such differences may possibly be due to cultural standards, palliation measures, and healthcare infrastructure.

A recent study proposed that Bacillus Calmette-Guérin (BCG) vaccination provides some protection against viral disease. This vaccine has been utilized since the early 1920s; it is a safe vaccine that showed safeguarding against a variety of pathogens and viruses [4]. The association between BCG vaccination and reduction in COVID-19 severity that is consistently being observed may currently be insufficient to demonstrate a causal link between BCG vaccine and protection against COVID-19 infection. However, ongoing research is currently trying to determine BCG vaccination roles in containing and inferring protection against COVID-19 mortality and morbidity and may have impacted the prevalence patterns seen in some countries. If this theory holds true, it can lead to beneficial connotations regarding worldwide vaccination regimens. Its possible transient protection and unspecified immune system enhancement can be particularly useful in vulnerable populations as well as help in buying time while waiting for the availability of COVID-19 specific vaccines and other treatments [5]. It is imperative to study the regional differences in prevalence and mortality patterns with possible exposure parameters such as BCG vaccination that can give 


\section{Cureus}

us insight into COVID-19 preventative measures and better preparedness for future epidemics.

\section{Materials And Methods}

This review study was conducted using standard review methods. The main study objective was to report the mean prevalence of COVID-19 infection across 20 countries. Furthermore, this study also performed a comparative analysis between countries with established current BCG vaccination policies and those without such a policy. This was done using a two-tailed t-test. Data were represented using mean values, standard deviation, and standard mean error. All statistical analysis and calculations were conducted using GraphPad (GraphPad Software Inc., California, USA). All Figures were generated using ChartGo software. COVID-19 related statistics were extracted from worldometers (https://www.worldometers.info/coronavirus/) according to the update on September 5, 2020 [6]. The vaccination status of each country was taken from the World Health Organization (WHO) BCG immunization report $[7,8]$.

\section{Results}

The analysis involved 20 countries. Characteristics included in the study are outlined in Tables $1-2$. Table 3 represents the comparative analysis of COVID-19 prevalence in countries with BCG vaccination and countries without BCG vaccination as a policy. The statistical difference of COVID-19 prevalence in countries with BCG vaccination and countries without BCG vaccination as a policy was observed to have a mean difference of -189348.70 (95\% CI: 414371.66 to 35674.26 ). There was a statistically insignificant difference in COVID-19 prevalence when comparing countries with a BGC policy and countries without it $(\mathrm{P}>0.05 ; \mathrm{P}=0.08)$.

\begin{tabular}{|c|c|c|c|c|}
\hline Country & Population & Total cases & Total deaths & Case fatality rate \\
\hline 1.India & 1369 Million & $4,020,239$ & 69,635 & $1.7 \%$ \\
\hline 2.Pakistan & 220.8 Million & 297,512 & 6,335 & $2.1 \%$ \\
\hline 3.Brazil & 210 Million & $4,091,801$ & 125,584 & $3.2 \%$ \\
\hline 4.Japan & 126.9 Million & 70,268 & 1,330 & $1.9 \%$ \\
\hline 5.Ireland & 5 Millions & 29,303 & 1,777 & $6 \%$ \\
\hline 6.Ecuador & 17.50Millions & 117,175 & 6,674 & $5.7 \%$ \\
\hline 7.Thailand & 67.79Milions & 3,431 & 58 & $1.7 \%$ \\
\hline 8. Saudi & 34.14Million & 319,141 & 4,015 & $1.2 \%$ \\
\hline 9.Indonesia & 257 Million & 187,537 & 7,832 & $4.2 \%$ \\
\hline 10.Chile & 17.4Million & 418,469 & 11,494 & $2.7 \%$ \\
\hline
\end{tabular}

TABLE 1: Countries that follow a national Bacillus Calmette-Guérin (BCG) vaccination policy and number of COVID-19 cases recorded 


\section{Cureus}

\begin{tabular}{|c|c|c|c|c|}
\hline Country & Population & Total cases & Total deaths & Case fatality rate \\
\hline 1.USA & 329.45 Million & $6,389,057$ & 192,111 & $3 \%$ \\
\hline 2.Italy & 60.3 Million & 274,644 & 35,518 & $13.1 \%$ \\
\hline 3.Spain & 47.1 Million & 517,133 & 29,418 & $6.1 \%$ \\
\hline 4.Germany & 83.02 Million & 250,281 & 9,401 & $3.8 \%$ \\
\hline 5.France & 67.0 Million & 309,156 & 30,724 & $10 \%$ \\
\hline 6.Austria & 9.01 Million & 28,729 & 735 & $3 \%$ \\
\hline 7.Belgium & 11.23 Million & 86,544 & 9,899 & $12 \%$ \\
\hline 8.UK & 66.4 Million & 342,351 & 41,537 & $13 \%$ \\
\hline 9.Switzerland & 8.48 Million & 43,532 & 2,013 & $5.3 \%$ \\
\hline 10.Norway & 5.42 Million & 11,231 & 264 & $2.8 \%$ \\
\hline
\end{tabular}

TABLE 2: Countries that did not have or ceased national Bacillus Calmette-Guérin (BCG) vaccination policy and their recorded cases

\begin{tabular}{|c|c|c|c|}
\hline & Mean of total current cases (Per 100,000 ) & Standard Deviation & Standard Error Mean \\
\hline Countries with BCG Vaccine Policy $(\mathrm{N}=10)$ & $9,55,488$ & 1639640.22 & 1800885 \\
\hline Countries without BCG Policy $(\mathrm{N}=10)$ & $8,25,266$ & 1961802.67 & 3188913 \\
\hline
\end{tabular}

TABLE 3: Comparative analysis between COVID-19 prevalence in countries with BCG vaccination policy and countries without Bacillus Calmette-Guérin (BCG) vaccine policy (per 100,000)

\section{Discussion}

The lower than expected COVID-19 cases in countries with poor health care systems is surprising. Statistical data on BCG vaccination policies by country may show a potential impact on the transmission of COVID-19 [9]. Our study demonstrated an insignificant relationship between COVID-19 prevalence and BCG regulations. The lowest prevalence was seen in Thailand, Indonesia, and Norway while the highest case numbers were seen in the United States (USA), Italy, and, Spain. Overall, in countries with proven BCG vaccine protocols in place, there was an insignificantly lower number of COVID-19 cases compared to those countries without such protocols $(\mathrm{P}>0.05)$ The country hardest hit by this infection is the USA.

Many nations have adopted compulsory BCG vaccination protocols for prophylaxis of tuberculosis (TB) over the past few decades which at the time was a major threat [10]. The majority of countries subsequently agreed to continue the policy, at least not too long ago (China, Ireland, Finland, and France). Other regions decided to cease the policy when TB was no longer a threat (Australia Spain, Ecuador). There were some countries that did not warrant a policy at all (USA, Italy, Lebanon). Although Iran has a current BCG vaccination policy, it can be convinced that these vaccine strains produce short-lived immunity compared to other countries widely used older strains [11]. Consequently, there are adequate variations in BCG vaccination policy, either its presence or absence throughout various districts around the globe, making it feasible to derive such a correlation.

The results obtained from this study are similar to a report by the World Health Organization stating "there is no evidence that the BCG vaccine protects people against infection with COVID-19 virus." [12] WHO discourages vaccination as a means of prophylaxis against COVID-19 until further evidence is presented. Although there is evidence of the nonspecific immune protective effects, these benefits have not been fully characterized. Furthermore, a study by Hensel et al. [13] also showed a lack of significance when comparing COVID-19 incidence in countries with a BCG vaccine policy and countries without a current BCG vaccine regimen. They concluded that the observation of incident differences could be connected to population density, age, incidence of TB, regional population, COVID-19 testing rates interlinked with BCG protocols causing confounding effects. On the contrary, a study conducted by Gursel and Gursel [14] showed that case 
numbers in countries that have BCG vaccinate programs were significantly less than those without a vaccine program $(\mathrm{P}<0.0001)$. In addition, Hegarty et al. [15] and Mahase [16] considered a link between national BCG vaccine protocols and mortality rates. They concluded that nations with a population-wide BCG program appear to show a lower number of cases and deaths due to COVID-19 infection, possibly due to the confirmed immunological benefits of the vaccination. Shet et al. [17] also reported a pattern of COVID-19related mortality amidst BCG-employed nations that was 5.8 times lower (95\% CI 1.8-19.0) compared to countries without a vaccination program. The discrepancies in these studies further highlight the difficulty of obtaining valid statistical data and indicates a need for further research on this comparison.

In general, a vaccine will provide its protection from distinct pathogens by its effector mechanism. A live attenuated vaccine such as BCG, an attenuated strain of Mycobacterium bovis, provides protection to pathogens causing respiratory infections $[18,19]$. There is enduring evidence that this vaccine induces unspecified mortality protection, the most commonly administered vaccine worldwide, enticing approximately $38 \%-45 \%$ reduction in fatality rates [20,21]. Developed to combat TB, its mortality benefits stem from a reduction in neonatal sepsis and respiratory infections. The unspecified immune advantages of BCG have been recognized as early as 1970 at the time when BCG showed to enhance immunity against pathogens such as listeria and influenza in mice models [22,23]. The underlying mechanism for this defense caused by the BCG vaccination is thought to be mediated through the activation of innate immune memory, or trained immunity [24]. In a randomized placebo controlled human study conducted by Arts et al. [25], it was evident that BCG vaccine induces genetic reprogramming of monocytes and shown protection against few experimental viral infections with an underlying key role of interleukin-1 beta (IL-1及) as a trained immune response. It can be contemplated that countries which continue BCG immunization can help in reducing the spread of COVID-19 better as compared to the countries which do not have BCG vaccination policies, although this is only speculation.

The comparative data from this study is acknowledged as being observational and predicated on single prevalence with the possibility of several confounding factors that can limit this study design. Since prevalence is dependent on testing capabilities in each region and may not represent the true degree of the epidemic regionally; a clear conclusion cannot be drawn due to such limited testing and reporting of cases [26]. A major challenge seen with the COVID-19 crisis has been obtaining clean data. The majority of available data related to COVID-19 has been of poor quality. This poor quality of comparable case data is greatly impacted by a nation's testing of the virus [27]. Therefore, the accuracy of prevalence, incidence, and other statistical values is of concern, limiting the validity of epidemiologic studies such as this. Nonetheless, during a pandemic with a rapid and exponentially growing disease as COVID-19, epidemiologic studies can provide beneficial insight into public health policies and crisis management.

\section{Conclusions}

A clear variance in COVID-19 prevalence is seen between the Western and Eastern hemispheres. There is conjecture that BCG vaccines' protective activity may be linked to lower numbers of cases in Asian and African countries. Although this study showed inconclusive results, the validity of this hypothesis can only be concluded by comparing the prevalence and deaths from BCG vaccinated and unvaccinated countries throughout the entire duration of this pandemic. Future studies on BCG vaccination may provide better understanding on vulnerable populations and prevention efforts.

\section{Additional Information \\ Disclosures}

Human subjects: All authors have confirmed that this study did not involve human participants or tissue. Animal subjects: All authors have confirmed that this study did not involve animal subjects or tissue. Conflicts of interest: In compliance with the ICMJE uniform disclosure form, all authors declare the following: Payment/services info: All authors have declared that no financial support was received from any organization for the submitted work. Financial relationships: All authors have declared that they have no financial relationships at present or within the previous three years with any organizations that might have an interest in the submitted work. Other relationships: All authors have declared that there are no other relationships or activities that could appear to have influenced the submitted work.

\section{Acknowledgements}

Dr. Sateesh Arja got the fellowship from the International Association of Medical Education in Europe (AMEE). The first one to receive in the Caribbean.

\section{References}

1. WHO: coronavirus disease 2019 (COVID-19) situation report - 87 . (2020). Accessed: April 24, 2020: https:/www.who.int/docs/default-source/coronaviruse/situation-reports/20200416-sitrep-87-covid-19.pdf.

2. Rajarshi K, Chatterjee A, Ray S: BCG vaccination strategy for prevention against COVID-19: hype or hope? Preprints. 2020, 10.20944/preprints202004.0351.v1

3. Miller A, Reandelar MJ, Fasciglione K, Roumenova V, Li Y, Otazu GH: Correlation between universal BCG 
vaccination policy and reduced morbidity and mortality for COVID-19: an epidemiological study. MedRxiv. 2020, 10.1101/2020.03.24.20042937

4. Daily mortality rates from COVID-19 classified by country BCG vaccine policy . (2020). Accessed: April 24, 2020: https://qxlab.ucd.ie/bcgcovid.pdf.

5. Escobar LE, Molin-Cruz A, Barillas-Mury C: BCG vaccine protection from severe coronavirus disease 2019 (COVID-19). Proc Natl Acad Sci U S A. 2020, 117:17720-17726. 10.1073/pnas.2008410117

6. Worldometer: coronavirus update (Live). (2020). Accessed: April 24, 2020: https://www.worldometers.info/coronavirus/.

7. WHO: report on BCG vaccine use for protection against mycobacterial infections including tuberculosis, leprosy, and other nontuberculous mycobacteria (NTM) infections. (2020). Accessed: April 24, 2020: https://www.who.int/immunization/sage/meetings/2017/october/1_BCG_report_revised_version_online.pdf.

8. Zwerling A, Behr MA, Verma A, Brewer TF, Menzies D, Pai M: The BCG World Atlas: a database of global BCG vaccination policies and practices. PLoS Med. 2011, 8:e1001012. 10.1371/journal.pmed.1001012

9. Sala G, Miyakawa T: Association of BCG vaccination policy with prevalence and mortality of COVID-19 . MedRxiv. 2020, 10.1101/2020.03.30.20048165

10. Berg MK, Yu Q, Salvador CE, Melani I, Kitayama S: Mandated Bacillus Calmette-Guérin (BCG) vaccination predicts flattened curves for the spread of COVID-19. MedRxiv. 2020, 10.1101/2020.04.05.20054163

11. Hu Y. Chen Y, Liang H, Wang Y: An overview of coverage of BCG vaccination and its determinants based on data from the coverage survey in Zhejiang province. Int J Environ Res Public Health. 2018, 15:1155. 10.3390/ijerph15061155

12. WHO: Bacille Calmette-Guérin (BCG) vaccination and COVID-19. (2020). Accessed: April 24, 2020: https://apps.who.int/iris/bitstream/handle/10665/331745/WHO-2019-nCoV-Sci_Brief-BCGvaccination2020.1-eng.pdf.

13. Hensel J, McAndrews JM, McGrail DJ, Dowlatshahi DP, LeBleu VS, Kalluri R: Exercising caution in correlating COVID-19 incidence and mortality rates with BCG vaccination policies due to variable rates of SARS CoV-2 testing. MedRxiv. 2020, 10.1101/2020.04.08.20056051

14. Gursel M, Gursel I: WITHDRAWN: Is global BCG vaccination coverage relevant to the progression of SARSCoV-2 pandemic?. Med Hypotheses. 2020, 10.1016/j.mehy.2020.109707

15. Hegarty P, Kamat AM, Zakirakis H, Dinardo A: BCG vaccination may be protective against Covid-19 [Epub ahead of print]. Research Gate. 2020, 10.13140/RG.2.2.35948.10880

16. Mahase E: Covid- 19: what treatments are being investigated?. BMJ. 2020, 368:m1252. 10.1136/bmj.m1252

17. Shet A, Ray D, Malavige N, Santosham M, Bar-Zeev N: Differential COVID-19-attributable mortality and BCG vaccine use in countries. MedRxiv. 2020, 10.1101/2020.04.01.20049478

18. Roth A, Gustafson P, Nhaga A, et al.: BCG vaccination scar associated with better childhood survival in Guinea-Bissau. Int J Epidemiol. 2005, 34:540-547. 10.1093/ije/dyh392

19. Hollm-Delgado MG, Stuart EA, Black RE: Acute lower respiratory infection among Bacille Calmette-Guérin (BCG)-vaccinated children. Pediatrics. 2014, 133:e73-e81. https://doi.org/10.1542/peds.2013-2218

20. Biering-Sørensen S, Aaby P, Lund N, et al.: Early BCG-Denmark and neonatal mortality among infants weighing <2500 g: a randomized controlled trial. Clin Infect Dis. 2017, 65:1183-1190. 10.1093/cid/cix525

21. Garly ML, Martins CL, Balé C, et al.: BCG scar and positive tuberculin reaction associated with reduced child mortality in West Africa: a non-specific beneficial effect of BCG?. Vaccine. 2003, 21:2782-2790. 10.1016/s0264-410x(03)00181-6

22. Spencer JC, Ganguly R, Waldman RH: Nonspecific protection of mice against influenza virus infection by local or systemic immunization with Bacille Calmette-Guerin. J Infect Dis. 1977, 136:171-175. 10.1093/infdis/136.2.171

23. Ratzan KR, Musher DM, Keusch GT, Weinstein L: Correlation of increased metabolic activity, resistance to infection, enhanced phagocytosis, and inhibition of bacterial growth by macrophages from Listeria- and BCG-infected mice. Infect Immun. 1972, 5:499-504.

24. Netea MG, Quintin J, van der Meer JW: Trained immunity: a memory for innate host defense . Cell Host Microbe. 2011, 9:355-361. 10.1016/j.chom.2011.04.006

25. Arts RJW, Moorlag SJCFM, Novakovic B, et al.: BCG vaccination protects against experimental viral infection in humans through the induction of cytokines associated with trained immunity. Cell Host Microbe. 2018, 23:89-100. 10.1016/j.chom.2017.12.010

26. Green CM, Fanucchi S, Fok ET, et al.: COVID- 19: a model correlating BCG vaccination to protection from mortality implicates trained immunity. MedRxiv. 2020, 10.1101/2020.04.10.20060905

27. Coronavirus disease (COVID-19) - statistics and research . (2020). Accessed: April 24, 2020: https://ourworldindata.org/coronavirus. 